\title{
Professor Erich Lehmann's Contributions to Testing of Hypotheses and Estimation
}

\author{
C.R. Rao
}

Professor Erich Lehmann, a major figure in the field of statistics, made fundamental contributions to several areas of statistical theory, especially in testing of hypotheses and estimation of unknown parameters. Although the foundations of these statistical methods were laid by Fisher, Neyman and Wald during the first half of the last century, no critical evaluation of these techniques was available. Lehmann by himself and in collaboration with some of his colleagues at the statistics department of the University of California, Berkeley, made a systematic study of these problems and provided a coherent theoretical structure. His contributions are reflected in his two great books. Testing of Hypotheses (1959) and Theory of Point Estimation (1983), which continue to be center pieces of graduate statistical education and have been translated into several languages.

The concepts of similar regions and sufficient statistics were introduced at an early stage in the development of statistical theory and both have proved extremely fruitful. A comprehensive discussion of these concepts was given in papers [4] and [6], making appropriate modifications and extending their use to a wide variety of problems in testing of hypotheses and estimation of parameters. The concept of minimal sufficient statistic was introduced to ensure optimality of certain procedures. A justification was given for the restriction to functions of sufficient statistic in testing of hypotheses similar to Rao-Blackwellization in estimators. The theory of completeness, similar regions and unbiased estimation was developed in greater generality than is customary in statistical papers. In [5], the uniqueness of unbiased estimators with uniformly minimum variance in sequential sampling was explored with special reference to Poisson, rectangular and binomial distributions. These three papers constitute a final authority on various issues related to hypotheses testing and unbiased estimation.

\footnotetext{
C.R. Rao

Professor Emeritus of Statistics, Eberly Professor Emeritus, Department of Statistics, Penn State University, University Park, PA 1602

e-mail: crr1@psu.edu
} 
Situations where unbiased estimators do not exist or do not seem to be reasonable were discussed in [9]. Several examples were given where unbiased estimators do not exist or seem to be unsatisfactory. The same situation arises with maximum likelihood (ML) estimation when the sample does not contain sufficient information. In such cases ML estimators may be subject to large bias which make them not useful for practical applications. It is an open problem whether alternative methods of estimation exist in cases where reasonable minimum variance unbiased estimators or ML estimators do not exist.

Two problems of interest on unbiased estimation in convex families of distributions were considered in [2]. Let $X_{1}, \ldots, X_{n}$ be an i.i.d. sample from some distribution $F \in \mathbb{F}$, a family of distributions. Two problems of interest discussed in the earlier literature are as follows:

Problem 1: If a functional $q$ is given on $\mathbb{F}$, does there exist a statistic $\delta\left(X_{1}, \ldots, X_{n}\right)$ such that $E(\delta \mid F)=q(F)$ for all $F \in \mathbb{F}$ or in other words, when does there exist an unbiased estimate of $q(F)$ based on a sample of $n$ observations?

Problem 2: The degree of a functional $q$ is defined as the smallest sample size $n \leq$ $\infty$ such that an unbiased estimator $\delta$ exists for $q$.

Satisfactory solutions were obtained for these two problems when $\mathbb{F}$ is closed under finite mixtures (convex combinations). Applications of these results were considered in a number of cases.

A minimax property of the sample mean in finite populations was proved in [3], under some conditions. Let $A=\left(a_{1}, \ldots, a_{n}\right)$ be the population and $\left(y_{1}, \ldots, y_{n}\right)$ be an estimate of $\bar{a}$, where $y_{1}, \ldots, y_{n}$ are i.i.d. observations from $A$. Further let $\tau^{2}=$ $\left(a_{i}-\bar{a}\right)^{2}$. In an earlier paper, [1], it was proved that

$$
\sup E(\delta-\bar{a})^{2} / \tau^{2}
$$

is minimized when $\delta=\bar{y}=\left(y_{1}+\cdots+y_{n}\right) / n$. It was proved in [3] that

$$
\sup E(\delta-\bar{a})^{2}
$$

is a minimum when $\delta=\bar{y}$ under the conditions $\tau^{2} \leq M$. It was further shown that the same result holds if the sample includes information on the labels of the sample units, and also under stratified sampling.

A comprehensive discussion of ancillary and sufficient statistics, and the concepts of completeness and bounded completeness was given in [8]. Basu proved that when a sufficient statistic $T$ is boundedly complete, then all ancillary statistics are independent of $T$. The converse of Basu's theorem that independence of all ancillary statistics from a sufficient statistic implies that $T$ is boundedly complete is not true. Some alternative definitions of ancillarity and completeness were given in [8] which imply the converse of Basu's theorem.

Nonparametric tests were introduced quite early in the development of statistics by Pitman, Willcoxon, Kruskal, Wallis and others. In [7], an appropriate methodology was developed to obtain point estimates of location or shift parameters of a distribution using rank test statistics. This seems to be an attractive area where further research is necessary. 
Professor Erich Lehmann's Contributions to Testing of Hypotheses and Estimation

\section{References}

[1] Aggarwal, O. P. (1955). Bayes and minimax procedures in sampling from finite and infinite populations-I. Ann. Math. Statist., 30, 206-218.

[2] Bickel, P. J. and Lehmann, E. L. (1969). Unbiased estimation in convex families. The Annals of Mathematical Statistics, 40, 1523-1535.

[3] Bickel, P. J. and Lehmann, E. L. (1981). A minimax property of the sample mean in finite populations. The Annals of Statistics, 9, 119-122.

[4] Hodges, Jr. J. L. and Lehmann, E. L. (1963). Estimation of location based on rank tests. The Annals of Mathematical Statistics, 34, 598-611.

[5] Lehmann, E. L. and Scheffe, H. (1950). Completeness, similar regions and unbiased estimation part 1. Sankhya, 10, 305-340.

[6] Lehmann, E. L. and Scheffe, H. (1956). Completeness, similar regions and unbiased estimation part 2. Sankhya, 15, 219-236, 17, 250.

[7] Lehmann, E. L. and Stein, C. (1950). Completeness in the sequential case. The Annals of Statistics, 21, 376-385.

[8] Lehmann, E. L. (1981). An interpretation of completeness and Basu's theorem. Journal of the American Statistical Association, 76, 335-340.

[9] Lehmann, E. L. (1983). Estimation with inadequate information. Journal of the American Statistical Association, 78, 624-627. 